\title{
Impact of the next-generation sequencing data depth on various biological result inferences
}

\author{
HOU Rui, YANG ZhenXing, LI MingHui \& XIAO HuaSheng* \\ National Engineering Center for Biochip at Shanghai, Shanghai 201203, China \\ Received December 29, 2012; accepted January 8, 2013
}

\begin{abstract}
Next-generation sequencing (NGS) technologies have revolutionized the field of genomics and provided unprecedented opportunities for high-throughput analysis at the levels of genomics, transcriptomics and epigenetics. However, the cost of NGS is still prohibitive for many laboratories. It is imperative to address the trade-off between the sequencing depth and cost. In this review, we will discuss the effects of sequencing depth on the detection of genes, quantification of gene expression and discovering of gene structural variants. This will provide readers information on choosing appropriate sequencing depth that best meet the needs of their particular project.
\end{abstract}

next-generation sequencing, depth, gene discovery, gene expression, structure variation

Citation: Hou R, Yang Z X, Li M H, et al. Impact of the next-generation sequencing data depth on various biological result inferences. Sci China Life Sci, 2013, 56: 104-109, doi: 10.1007/s11427-013-4441-0

The capillary electrophoresis-based Sanger sequencing method [1] of sequencing by capillary electrophoresis is considered as a 'first-generation' technology, which has been employed in many whole genome sequencing projects and is considered as the 'gold standard' in terms of both read length and sequencing accuracy. In the relatively short time frame since 2005, several next-generation sequencing (NGS) technologies (also known as massively parallel sequencing) have emerged, including Roche 454, Illumina Hiseq 2000, ABI SOLiD, as well as single molecule sequencing technology of Helicos Biosciences and Pacific Biosciences [2], which could make it possible for even single research groups to generate large amounts of sequence data very rapidly and at a substantially lower cost than Sanger method on the ABI 3730xl platform. The significant advances in NGS are revolutionizing the field of genomics and provided unprecedented opportunities for highthroughput analysis at the level of genomics, transcriptom-

*Corresponding author (email: huasheng_xiao@shbiochip.com) ics and epigenetics.

NGS technologies have increased high-throughput capacity and reduced the cost, which makes it possible to use these new platforms for de novo sequencing of entire genomes of many species. To date, whole-genome sequencing of many non model species from microbes, plants to animals are available [3-5], facilitated by NGS approaches. For those organisms with a previously published reference genome sequence, NGS can be applied to their wholegenome resequencing [6] for variant discovery, such as single-nucleotide polymorphisms (SNPs), insertions/deletions (InDels), and structural rearrangements. Besides, methods like RRL [7] and RAD [8,9] also allow for discovery of mutations in any non model organisms that are lack of genomic information. Apart from whole genome sequencing, there is much more interest in applying NGS platforms, coupled with DNA target capture technology [10] for focused analysis of specific genomic regions, such as intervals identified through single nucleotide polymorphism (SNP)based association or linkage studies, candidate genes or the 
whole exome.

In addition to DNA sequencing, the massively parallel sequencing can be applied to sequence RNA, known as RNA-Seq [11]. Microarrays [12] have been recognized as the predominant method for large-scale studies at gene expression levels in past years. In comparison, RNA-Seq is a more powerful tool for comprehensive characterization of whole transcriptome at both gene and exon levels and with an additional ability to identify rare transcripts, new genes, novel splicing junctions and gene fusions. And because RNA-Seq approach does not require the knowledge of the genome sequence as a prerequisite, non model organisms could obtain transcripts information on the sequence level. Furthermore, analysis of small RNAs and other noncoding RNAs with massively parallel sequencing are likely to elucidate the roles of those molecules in gene regulation [13].

Epigenetics is to study heritable gene regulation that does not involve the DNA sequence. Modified NGS protocols also allow for an unbiased assessment of DNA/histone modifications and DNA-protein interactions. The genomewide single-base resolution of DNA methylation mapping has been performed using bisulfate sequencing [14,15]. Chromatin modifying protein binding can be mapped using chromatin immunoprecipitation sequencing (ChIP-Seq) [16].

The cost of NGS is still prohibitive for many laboratories. NGS platforms allow adding sample specific barcode (sometimes called index) to the library molecules. Then it would be cost-effective if multiple samples are multiplexed and sequenced in a single lane with appropriate sequencing depth. Therefore, one of the most important concerns about a particular NGS experiment is the requirement of sequencing depth. Here, we will discuss the effects of sequencing depth on the detection of genes, quantification of gene expression and discovering of structural variants. This will provide readers information on choosing appropriate sequencing depth that best suit the needs of their particular research.

\section{Effect of sequencing depth on gene discovery}

RNA-Seq has become a popular method for extracting the transcriptome information. The more the target is sequenced, the more genes are identified. This will presumably result in a more accurate estimation of the expression level. As a consequence, the ability to find genes and detect differential expression is determined by sequencing depth. Low sequencing depth only allows the detection of highly expressed genes. Deeper sequencing is required for those transcripts that are expressed at lower levels. This leads to the question of how many reads should be sufficient to cover the transcriptome of a given sample.

To address this question, we randomly sampled 1-116 million reads from the total reads of three human samples
(Figure 1). The number of genes detected was increasing rapidly, and reached the turning point at 20 million reads, with about $45 \%$ of ENSEMBL genes were identified. The gene number increased much slowly when the read size was larger than 20 million. The saturation plots were almost identical across the data from sample A and B. In comparison, sample $\mathrm{C}$ needed more reads to reach the same percentage of gene detected. The main reason behind the difference was the different RNA preparation method. Sample $A$ and B used poly-A mRNA for sequencing while sample $\mathrm{C}$ used the total RNA with rRNA depletion. Non-coding RNA in sample $C$ therefore has a clear influence in the relative proportion of protein coding genes.

Similarly, Huang et al. RNA-Seq analyses for 10 matched pairs of cancer and normal tissues from $\mathrm{HBV}$-related hepatocellular carcinoma (HCC) patients [17]. They randomly sampled 5-65 million reads from the total raw reads from sample A39P and A39C separately. About 15 million raw reads were sufficient to identify more than $50 \%$ ENSEMBL genes. This number almost equalled to all detectable expressed genes in a given human tissue. Another research [18] divided their 879 million 50 bp read data from RNA-Seq of cultured human B-cells set into smaller sets and analyzed how the detection of a gene varies with increasing sequencing depth. The total 879-million-read data was assumed to give a comprehensive catalog of transcribed genes. Then the number of genes detected was assessed in fractions of total reads (final data). The number of genes detected reached a plateau at 50 million reads, with about $75 \%$ of genes detected. With 100 million reads, $81 \%$ of genes were detected. For each additional 100 million reads, $3 \%$ more genes were able to be detected on average. Furthermore, expression level of genes also affects the number of genes detected. With 100 million reads, $80 \%$ of highly expressed genes (top 25th percentile) compared to $32 \%$ of the low expression genes (bottom 25th percentile) were detected.

Additional genes were able to be detected with more reads. However, once the saturation curve excess the turning point, increment of reads results in only a small number of additional genes. To address the trade-off between the

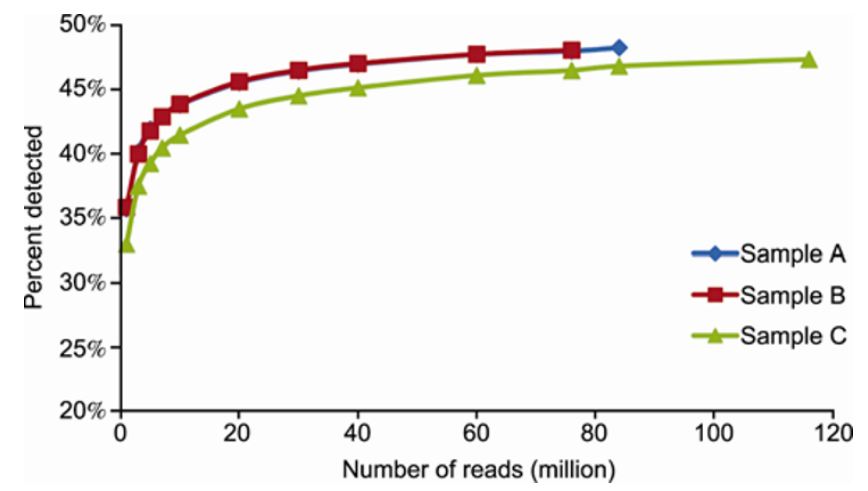

Figure 1 Number of genes detected at different sequencing depths. 
depth of RNA-Seq and the coverage of the transcriptome in an organism, Tarazona et al. [19] used new detections rate (NDR), the number of newly detected genes in 1 million additional reads, as a function of the sequencing depth for each of the three published data. Although all three experiments saturation is not entirely reached, each data set has a different sequencing depth; NDRs at the highest depth are substantially different. The NDR value dropped from 232 to 70 , and then to 19 , with 22,45 and 200 million reads respectively. In addition, NDR values are broadly similar across data sets for a given number of reads, suggesting that these saturation curves could be indicative for other human data sets generated by Illumina platform.

\section{Effect of sequencing depth on quantify gene expression}

If only the number of genes was concerned, 15 or 50 million reads could be an answer according to the above discussion. However, for most studies, accurate estimating of expression level is more important. Toung's data [18] gave us a good advice. The expression values assessed by total 879 million reads were set to be the final levels, the sequencing depth necessary to achieve these final levels was analyzed then. At least 400 million reads are needed, when $50 \%$ of the genes are required to be within $10 \%$ of their final measurements. This is a too large number for most researchers.

If we perform a RNA-Seq experiment using pair-end sequencing $(2 \times 100 \mathrm{bp})$ approach with $4 \mathrm{G}$ per sample (equals to $20 \mathrm{M}$ reads in single-end sequencing like $1 \times 50 \mathrm{bp}$ ). With this popular depth for most studies at present, only $1 \%-2 \%$ of genes will have values that are within $10 \%$ of their final value. Even with 100 million reads, $6 \%$ of genes are within this margin of error. Furthermore, although 100 million reads is sufficient for detection of the majority of genes (72\%), the expression levels of these genes deviate from their final value by $41 \%$ on average. In other words, if we only consider those highly expressed genes $(50 \%$ of the total number), and we require these genes to be within $20 \%$ of the final measurements, about 170 million reads are needed. Another two studies by Mortazavi et al. [20] and $\mathrm{Xu}$ et al. [21] also illuminated that deeper sequencing depth was required for more accurate estimation of low expression genes.

\section{Effect of sequencing depth on structural var- iant discovery}

Sequencing depth directly affects the accuracy of expression level estimation, and also affects the structural variant discovery. Alternative splicing is an essential mechanism for increasing transcriptome plasticity and proteome diversity in eukaryotes. About $95 \%$ of human genes have alternative splicing, evaluated by a high-throughput sequencing results [22]. As a consequence, the number of splice junctions detected in an experiment close relate to sequencing depth just as the number of genes do. With 100 million reads, $90 \%$ of transcripts and $76 \%$ of splicing junctions were detected (Figure 1). For each additional 100 million reads, $1 \%$ more transcripts and $4 \%$ more junctions were able to be detected on average. Furthermore, sufficient sequence coverage is also necessary to estimate the expression of different spliced isoforms and determine their relative abundance. At deficient sequencing depth, low expressed isoforms have large deviation from the final level. What is even worse that the relative abundance between isoforms could be misjudged in some situation [18].

The relative high false positive rate of variants such as SNVs and InDels discovered by RNA-Seq is still a problem [23]. In comparison, whole exome capture sequencing is one of the most frequency applied technique in SNV discovering and subsequently identification of disease-associated alleles. The average depth has increased about three times from this method been applied. We have reviewed 29 publications of whole exome sequencing (Table 1), and found the common depth of exome seqencing was about 30-40x two years ago and about 60-100× recently.

The depth bias in different exon area is the major problem encountered in exome seqencing, as show in Figure 2, about $20 \%$ exon region are less than $20 \times$ when the average depth is about $50 \times$. The lower the depth is, the higher FDR would exist in SNV and InDel detection. Allele bias is a neglected problem caused by different hybridization efficiency for reference sequence and mutant sequence. The mutant fragment has less opportunity than the right sequence which is perfect match of the probe. It is difficult to calculate the reference-bias for the effect of GC content and hybridization Tm [53].

Allelic balance $(\mathrm{AB})$ was calculated by determining the ratio of reference base calls over the total number of calls at every SNV (AB=0.53-0.55) [54]. We apply ABR (Allele Bias Rate), $A B R=$ ref depth/alt depth, to study whether the bias of InDel is higher than SNV. We also calculate the change of SNV/InDel number and ABR along with depth increment using an exome data (186 $\mathrm{M}$ mapped reads) in order to estimate the proper depth sufficient (Figure 3). The result shows that the number of SNV in whole genome keep rising with the depth increment and much higher than SNV number in exon, which saturated at $10 \mathrm{G}$ mapped data, which means that the low depth region outside exon result in a lot of false positive. The InDel number in exon or whole genome saturated at $10 \mathrm{G}$ mapped data, which means that the accuracy of InDel in lower coveraged region is much higher than SNV.

ABR of InDel is higher than SNV because InDel forms loop structure and reduces the stability of the double strands. ABR of SNV and InDel change slightly from 1.2 to 1.3 
Table 1 Sequencing depth in publications ${ }^{\text {a) }}$

\begin{tabular}{|c|c|c|c|c|}
\hline Date & Sequencing platform & Capture platform & Depth/Sample & Reference \\
\hline Nov 2009 & GA II & Agilent $244 \mathrm{~K}$ microarrays & $40 x$ & {$[24]$} \\
\hline May 2010 & SOLiD & Agilent & $43 \times$ & {$[25]$} \\
\hline Apr 2011 & GA IIx $2 \times 76$ & Agilent & $16 \mathrm{G}, \sim 180 \times$ & {$[27]$} \\
\hline Jun 2011 & GA II $2 \times 78$ & Agilent & Two lanes (*about $10 \mathrm{G}, 100-130 \times$ ) & {$[28]$} \\
\hline Jul 2011 & GAII & Agilent $38 \mathrm{M}$ & $11.7-13.4 \mathrm{G}$ & {$[29]$} \\
\hline Aug 2011 & GA II $2 \times 75$ & Agilent $38 \mathrm{M}$ & $98 \times$ & {$[30]$} \\
\hline Aug 2011 & HiSeq $20002 \times 50$ & Agilent & $7.3 \mathrm{G}$ (*about $70-900 \times)$ & {$[31]$} \\
\hline Aug 2011 & GA II $2 \times 101$ & Agilent & One lane (*about 6-7 G, 60-80x) & {$[32]$} \\
\hline Dec 2011 & GA II & Agilent $50 \mathrm{M}$ & $\sim 65 \times$ & {$[33]$} \\
\hline Dec 2011 & GA IIx $2 \times 76$ & SeqCap EZ & $1.4-5.7 \mathrm{G}, 27-163 \times$ & {$[34]$} \\
\hline May 2012 & GA II+HiSeq 2000 & Agilent & $\sim 80 \times$ & {$[35]$} \\
\hline May 2012 & HiSeq & Agilent $38 \mathrm{M}$ & $118 \times$ & {$[37]$} \\
\hline Jun 2012 & SOLiD 4 & Agilent $50 \mathrm{M}$ & $1 / 4$ run & {$[38]$} \\
\hline Jun 2012 & HiSeq $20002 \times 100$ & Agilent $50 \mathrm{M}$ & $93 \%$ target $>30 \times$ & {$[39]$} \\
\hline Jul 2012 & GA II+HiSeq 2000 & Agilent $50 \mathrm{M}$ & $90 \times$ & {$[40]$} \\
\hline Jul 2012 & HiSeq $20002 \times 101$ & Agilent $50 \mathrm{M}$ & $30-35 \mathrm{G}$ & {$[41]$} \\
\hline Jul 2012 & SOLiD 4 & Agilent $50 \mathrm{M}$ & $43 \times$ & {$[42]$} \\
\hline Jul 2012 & GA II $2 \times 76$ & Nimblegen SeqCap EZ & $86 x$ & {$[43]$} \\
\hline Jul 2012 & HiSeq $20002 \times 76$ & NA & $103 \times$ & {$[44]$} \\
\hline Jul 2012 & GA IIx + HiSeq $20002 \times 76$ & Nimblegen SeqCap EZ & $65 \pm 14.8 \times$ & {$[45]$} \\
\hline Aug 2012 & HiSeq 2000 & Agilent $38 \mathrm{M}$ & $>100 \times(>11 \mathrm{G})$ & {$[46]$} \\
\hline Aug 2012 & GA II $1 \times 75+$ SOLiD & NimbleGen+Agilent & $28 \times, 88 \times$ & {$[47]$} \\
\hline Sept 2012 & GA II $2 \times 95$ & agilent $38 \mathrm{M}$ & $190 \times$ & {$[48]$} \\
\hline Sept 2012 & GA II $2 \times 75$ & Agilent $38 \mathrm{M}$ & $6.9 \mathrm{G}(*$ about $60-80 \times)$ & {$[51]$} \\
\hline Oct 2012 & HiSeq $20002 \times 100$ & Agilent & $63 x$ & {$[52]$} \\
\hline
\end{tabular}

a) *, Estimates.

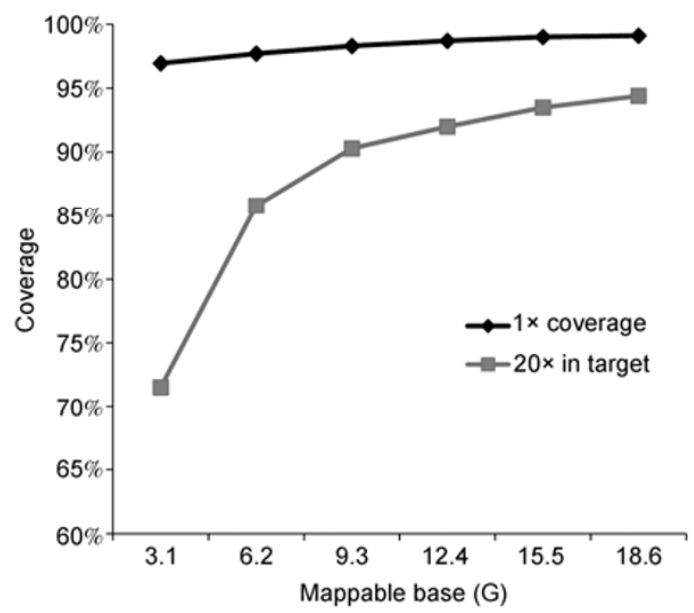

Figure 2 Coverage of $1 \times$ and $20 \times$ regions at different sequencing depths.

when depth increasing, which means that the bias is common but slight. The increment of InDel ABR may be caused by new InDels detected with lagre ABR when depth increasing. In summary, $10 \mathrm{G}$ of mapped data (average sequencing depth about $100 \times$ ) will be appropriate for exome seqencing, which matches the current trend.

\section{Conclusion}

In this study, we have discussed the effects of sequencing depth on detection of genes, quantification of gene expression and discovering of structural variants. The results indicated that although RNA-Seq effectively enhances our view on the diversity of the transcriptome, the quantification of true expression at a low depth might not be so easy to achieve. In practice, 15-50 million reads allow to detect the majority of genes in human tissue. However, at least 170 million reads are needed, when $50 \%$ of the genes were required to be within $20 \%$ of their final measurements. For the detection of splicing junctions, similar results were reached. For variants such as SNVs and InDels, exome se- 

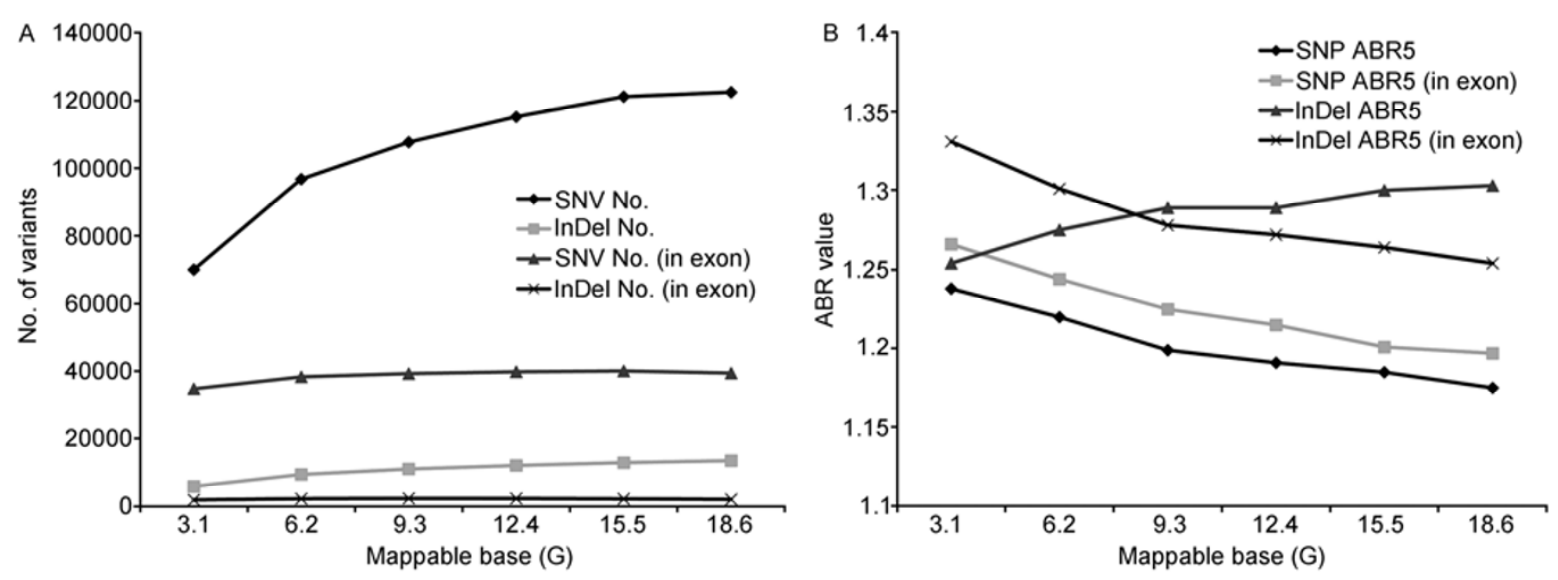

Figure 3 Number of varients (A) and ABR value (B) at different sequencing depths.

quencing is a powerful approach, and $10 \mathrm{G}$ of mapped data (about $100 \times$ ) will be appropriate for most studies, which matches the current trend.

1 Sanger F, Nicklen S, Coulson A R. DNA sequencing with chainterminating inhibitors. Proc Natl Acad Sci USA, 1977, 74: 54635467

2 Metzker M L. Sequencing technologies-the next generation. Nat Rev Genet, 2010, 11: 31-46

3 Huang S, Li R, Zhang Z, et al. The genome of the cucumber, $C u$ cumis sativus L. Nat Genet, 2009, 41: 1275-1281

4 Li R, Fan W, Tian G, et al. The sequence and de novo assembly of the giant panda genome. Nature, 2010, 463: 311-317

5 Hernandez D, Francois P, Farinelli L, et al. De novo bacterial genome sequencing: millions of very short reads assembled on a desktop computer. Genome Res, 2008, 18: 802-809

6 Wheeler D A, Srinivasan M, Egholm M, et al. The complete genome of an individual by massively parallel DNA sequencing. Nature, 2008, 452: 872-876

7 van Tassell C P, Smith T P, Matukumalli L K, et al. SNP discovery and allele frequency estimation by deep sequencing of reduced representation libraries. Nat Methods, 2008, 5: 247-252

8 Baird N A, Etter P D, Atwood T S, et al. Rapid SNP discovery and genetic mapping using sequenced RAD markers. PLoS ONE, 2008, 3 : e3376

9 Wang S, Meyer E, McKay J K, et al. 2b-RAD: a simple and flexible method for genome-wide genotyping. Nat Methods, 2012, 9: 808810

10 Ng S B, Turner E H, Robertson P D, et al. Targeted capture and massively parallel sequencing of 12 human exomes. Nature, 2009, 461: 272-276

11 Wang Z, Gerstein M, Snyder M. RNA-Seq: a revolutionary tool for transcriptomics. Nat Rev Genet, 2009, 10: 57-63

12 Fodor S P, Rava R P, Huang X C, et al. Multiplexed biochemical assays with biological chips. Nature, 1993, 364: 555-556

13 Morin R D, O'Connor M D, Griffith M, et al. Application of massively parallel sequencing to microRNA profiling and discovery in human embryonic stem cells. Genome Res, 2008, 18: 610-621

14 Lister R, O'Malley R C, Tonti-Filippini J, et al. Highly integrated single-base resolution maps of the epigenome in Arabidopsis. Cell, 2008, 133: 523-536

15 Ordway J M, Budiman M A, Korshunova Y, et al. Identification of novel high-frequency DNA methylation changes in breast cancer. PLoS ONE, 2007, 2: e1314

16 Johnson D S, Mortazavi A, Myers R M, et al. Genome-wide mapping of in vivo protein-DNA interactions. Science, 2007, 316: 1497-1502
17 Huang Q, Lin B, Liu H, et al. RNA-Seq analyses generate comprehensive transcriptomic landscape and reveal complex transcript patterns in hepatocellular carcinoma. PLoS ONE, 2011, 6: e26168

18 Toung J M, Morley M, Li M, et al. RNA-sequence analysis of human B-cells. Genome Res, 2011, 21: 991-998

19 Tarazona S, Garcia-Alcalde F, Dopazo J, et al. Differential expression in RNA-seq: a matter of depth. Genome Res, 2011, 21: 22132223

20 Mortazavi A, Williams B A, McCue K, et al. Mapping and quantifying mammalian transcriptomes by RNA-Seq. Nat Methods, 2008, 5: 621-628

$21 \mathrm{Xu} \mathrm{W}$, Seok J, Mindrinos M N, et al. Human transcriptome array for high-throughput clinical studies. Proc Natl Acad Sci USA, 2011, 108: 3707-3712

22 Pan Q, Shai O, Lee L J, et al. Deep surveying of alternative splicing complexity in the human transcriptome by high-throughput sequencing. Nat Genet, 2008, 40: 1413-1415

23 Cirulli E T, Singh A, Shianna K V, et al. Screening the human exome: a comparison of whole genome and whole transcriptome sequencing. Genome Biol, 2010, 11: R57

$24 \mathrm{Ng} \mathrm{S} \mathrm{B}$, Buckingham K J, Lee C, et al. Exome sequencing identifies the cause of a mendelian disorder. Nat Genet, 2010, 42: 30-35

25 Hoischen A, van Bon B W, Gilissen C, et al. De novo mutations of SETBP1 cause Schinzel-Giedion syndrome. Nat Genet, 2010, 42: 483-485

26 Wang K, Kan J, Yuen S T, et al. Exome sequencing identifies frequent mutation of ARID1A in molecular subtypes of gastric cancer. Nat Genet, 2011, 43: 1219-1223

27 Wei X, Walia V, Lin $\mathrm{J} \mathrm{C}$, et al. Exome sequencing identifies GRIN2A as frequently mutated in melanoma. Nat Genet, 2011, 43: $442-446$

28 Comino-Mendez I, Gracia-Aznarez F J, Schiavi F, et al. Exome sequencing identifies MAX mutations as a cause of hereditary pheochromocytoma. Nat Genet, 2011, 43: 663-667

29 Albers C A, Cvejic A, Favier R, et al. Exome sequencing identifies NBEAL2 as the causative gene for gray platelet syndrome. Nat Genet, 2011, 43: 735-737

30 Li M, Zhao $\mathrm{H}$, Zhang $\mathrm{X}$, et al. Inactivating mutations of the chromatin remodeling gene ARID2 in hepatocellular carcinoma. Nat Genet, 2011, 43: 828-829

$31 \mathrm{Xu} \mathrm{B}$, Roos J L, Dexheimer P, et al. Exome sequencing supports a de novo mutational paradigm for schizophrenia. Nat Genet, 2011, 43: 864-868

32 Sloan J L, Johnston J J, Manoli I, et al. Exome sequencing identifies ACSF3 as a cause of combined malonic and methylmalonic aciduria. Nat Genet, 2011, 43: 883-886

33 Quesada V, Conde L, Villamor N, et al. Exome sequencing identifies recurrent mutations of the splicing factor SF3B1 gene in chronic 
lymphocytic leukemia. Nat Genet, 2011, 44: 47-52

34 Nikolaev S I, Rimoldi D, Iseli C, et al. Exome sequencing identifies recurrent somatic MAP2K1 and MAP2K2 mutations in melanoma. Nat Genet, 2011, 44: 133-139

35 Ong C K, Subimerb C, Pairojkul C, et al. Exome sequencing of liver fluke-associated cholangiocarcinoma. Nat Genet, 2012, 44: 690-693

36 Arboleda V A, Lee H, Parnaik R, et al. Mutations in the PCNAbinding domain of CDKN1C cause IMAGe syndrome. Nat Genet, 2012, 44: 788-792

37 Barbieri C E, Baca S C, Lawrence M S, et al. Exome sequencing identifies recurrent SPOP, FOXA1 and MED12 mutations in prostate cancer. Nat Genet, 44: 685-689

38 Wortmann S B, Vaz F M, Gardeitchik T, et al. Mutations in the phospholipid remodeling gene SERAC1 impair mitochondrial function and intracellular cholesterol trafficking and cause dystonia and deafness. Nat Genet, 2012, 44: 797-802

39 Lee J H, Huynh M, Silhavy J L, et al. De novo somatic mutations in components of the PI3K-AKT3-mTOR pathway cause hemimegalencephaly. Nat Genet, 2012, 44: 941-945

40 Heinzen E L, Swoboda K J, Hitomi Y, et al. De novo mutations in ATP1A3 cause alternating hemiplegia of childhood. Nat Genet, 2012, 44: $1030-1034$

41 Falk M J, Zhang Q, Nakamaru-Ogiso E, et al. NMNAT1 mutations cause Leber congenital amaurosis. Nat Genet, 2012, 44: 1040-1045

42 Harakalova M, van Harssel J J, Terhal P A, et al. Dominant missense mutations in ABCC9 cause Cantu syndrome. Nat Genet, 2012, 44: 793-796

43 Emond M J, Louie T, Emerson J, et al. Exome sequencing of extreme phenotypes identifies DCTN4 as a modifier of chronic Pseudomonas aeruginosa infection in cystic fibrosis. Nat Genet, 2012, 44: 886-889

44 Hodis E, Watson I R, Kryukov G V, et al. A landscape of driver mu- tations in melanoma. Cell, 2012, 150: 251-263

45 Krauthammer M, Kong Y, Ha B H, et al. Exome sequencing identifies recurrent somatic RAC1 mutations in melanoma. Nat Genet, 2012, 44: 1006-1014

46 Rademakers R, Baker M, Nicholson A M, et al. Mutations in the colony stimulating factor 1 receptor (CSF1R) gene cause hereditary diffuse leukoencephalopathy with spheroids. Nat Genet, 2012, 44: 200-205

47 Huang J, Deng Q, Wang Q, et al. Exome sequencing of hepatitis B virus-associated hepatocellular carcinoma. Nat Genet, 2012, 44: $1117-1121$

48 Peifer M, Fernandez-Cuesta L, Sos M L, et al. Integrative genome analyses identify key somatic driver mutations of small-cell lung cancer. Nat Genet, 2012, 44: 1104-1110

49 Rudin C M, Durinck S, Stawiski E W, et al. Comprehensive genomic analysis identifies SOX2 as a frequently amplified gene in small-cell lung cancer. Nat Genet, 2012, 44: 1111-1116

50 Rice G I, Kasher P R, Forte G M, et al. Mutations in ADAR1 cause Aicardi-Goutieres syndrome associated with a type I interferon signature. Nat Genet, 2012, 44: 1243-1248

51 Doyle A J, Doyle J J, Bessling S L, et al. Mutations in the TGF-beta repressor SKI cause Shprintzen-Goldberg syndrome with aortic aneurysm. Nat Genet, 2012, 44: 1249-1254

52 Barcia G, Fleming M R, Deligniere A, et al. De novo gain-of-function KCNT1 channel mutations cause malignant migrating partial seizures of infancy. Nat Genet, 2012, 44: 1255-1259

53 Bainbridge $\mathrm{M} \mathrm{N}$, Wang M, Burgess D L, et al. Whole exome capture in solution with $3 \mathrm{Gbp}$ of data. Genome Biol, 2010, 11: R62

54 Clark M J, Chen R, Lam H Y, et al. Performance comparison of exome DNA sequencing technologies. Nat Biotechnol, 2011, 29: 908914

Open Access This article is distributed under the terms of the Creative Commons Attribution License which permits any use, distribution, and reproduction in any medium, provided the original author(s) and source are credited. 